\title{
The effect of close binary evolution on the chemical enrichment of galaxies
}

\author{
Erwin De Donder and Dany Vanbeveren \\ Astrophysical Institute, Free University of Brussels, \\ Pleinlaan 2, B-1050 Brussels, Belgium
}

\begin{abstract}
During their various evolutionary phases, stars enrich the interstellar gas (ISG) with chemical elements. In our study we investigate the effect of binaries on the chemical enrichment of the ISG. To derive the true yields for a population of single and binary stars we use a Population Number Synthesis code which is described in detail in Vanbeveren et al. (1998). We account for the enrichment through stellar wind (SW), supernova explosion (SN) of single and binary stars including type Ia, planetary nebula $(\mathrm{PN})$, and through binary interaction (BI). When black hole (BH) formation occurs, it is assumed that all matter is swallowed by the hole. This happens for single stars with a mass larger than $25 \mathrm{M}_{\odot}$ and for interacting binary components heavier than $40 \mathrm{M}_{\odot}$. Type Ia $\mathrm{SNe}$ are treated according to the scenario of $\mathrm{Li} \&$ van den Heuvel (1997). We conclude that including binaries: (a) increases the production of helium, up to a factor $\sim 1.8$ for $100 \%$ binaries (note that the helium yield increases linearly with binary frequency); and (b) drastically changes the ratio of the relative helium to metallicity enrichment $d Y / d Z$ (even for $Z=0.02$ ) resulting in values higher than 3.5 for binary frequencies $\geq 40 \%$, which is in good agreement with the observations.
\end{abstract}

\section{References}

Li, X.D., van den Heuvel, E.P.J. 1997, A\&A 322, L9

Vanbeveren, D., De Donder, E., Van Bever, J., Van Rensbergen, W., de Loore, C. 1998, New Astronomy, 3, 443 